\title{
Affections Neuroméningées Au Cours Du VIH Dans Le Service Des Maladies Infectieuses Du CHU De Brazzaville : Prévalence Et Facteurs Associés Au Décès
}

\section{Ossibi Ibara Bienvenu Rolland Obengui}

Service des Maladies Infectieuses, CHU-Brazzaville CONGO Département de Médecine, Faculté des sciences de la santé, UMNG CONGO

\section{Damba Banzouzi Bébene \\ Ossou-Nguiet Paul Macaire}

Département de Médecine, Faculté des sciences de la santé, UMNG CONGO Service de Neurologie, CHU-Brazzaville CONGO

\section{Paka Herdan}

Service des Maladies Infectieuses, CHU-Brazzaville CONGO

\section{Boumandouki Paul Jean Claude}

Service des Maladies Infectieuses, CHU-Brazzaville CONGO

Département de Médecine, Faculté des sciences de la santé, UMNG CONGO

\section{Puruenhce Marie Francke}

Service des Maladies Infectieuses, CHU-Brazzaville CONGO

\section{doi: 10.19044/esj.2016.v12n33p177 URL:http://dx.doi.org/10.19044/esj.2016.v12n33p177}

\begin{abstract}
Goal . Neuromeningeal determine the prevalence of disease in PLHIV and identify factors associated with death.

Methodology. Retrospective study on descriptive and analytical referred cases hospitalized in the Infectious Diseases department during the period 1 January 2013 to 31 May 2015.

Results. One hundred and fifty patients included (10.5\% of admissions) with mean age of $41.1 \pm 11.8$ years (range 19 to 69 ) women ( $n=86,57.3 \%)$, sex ratio of 1,3 . were without jobs $(n=68 ; 45.3 \%)$, single $(n=96 ; 64 \%)$, heterosexually infected by HIV-1 (100\%). The average time of consultation was $19.2 \pm 1.3$ days. Fever $(n=99 ; 66.0 \%)$, disorders of consciousness $(n=$ $66 ; 44.0 \%)$ were the main reasons for consultations. Meningeal stiffness (40\%), focal signs ( $\mathrm{n}=62 ; 41,4 \%$ ) were the main signs consideration. CSF was clear $(\mathrm{n}=94 ; 62.7 \%)$, hyperproteinorachique (59.8\%), hypoglycorrachique (43.8\%). Examining the ink was positive in 39 cases
\end{abstract}


(34.8\%) The Gerh was positive in 35 patients .the CT abnormalities were dominated abscess $(\mathrm{n}=12 ; 15.4 \%)$.. Thirty four patients were on TDF + FTC + EFV regimen. The average hospital stay was $14.4 \pm 9.5$ days. Overall mortality was $83.2 \%$ mainly due to cerebral herniation $(n=28 ; 33.3 \%)$.

Conclusion. The prevalence and lethality of diseases neuromeningeal of HIV are high. The diagnosis delay and TNM influence the prognosis of patients; Hence the importance of early detection of HIV infection for its management to prevent immunosuppression.

Keywords: Neuromeningeal disorders, HIV prevalence, lethality, CHUBrazzaville

\section{Résumé}

Objectif. Déterminer la prévalence des affections neuroméningées chez les PvVIH et rechercher les facteurs associés au décès.

Méthodologie. Etude rétrospective à visée descriptive et analytique des dossiers cas hospitalisés dans le service de Maladies Infectieuses durant la période allant du 1erjanvier 2013 au 31 mai 2015.

Résultats. Cent cinquante dossiers inclus (10,5\% des admissions) d’âge moyen de 41,1 \pm 11,8 ans(extrême : 19 et 69 ans) de sexe féminin ( $\mathrm{n}=86$, $57,3 \%)$, sex-ratio de 1,3.Ils étaient, sans emplois ( $n=68$; 45,3\%), célibataires ( $n=96 ; 64 \%)$, contaminés hétéro sexuellement par le VIH1(100\%). Le délai moyen de consultation était de 19,2 $\pm 1,3$ jours. La fièvre $(n=99 ; 66,0 \%)$, les troubles de la conscience $(n=66 ; 44,0 \%)$ étaient les principaux motifs de consultations. La raideur méningée (40\%), les signes focaux ( $\mathrm{n}=62 ; \%)$ étaient les principaux signes d'examen. Le LCR était clair $(n=94 ; 62,7 \%)$, hyperproteinorachique (59,8\%), hypoglycorrachique (43,8\%).L'examen à l'encre de chine était positif dans 39 cas (34,8\%).La GERH était positive chez 35 patients .les anomalies scanographiques étaient dominées par les abcès $(n=12 ; 15,4 \%)$. Trente quatre patients étaient sous régime $\mathrm{TDF}+\mathrm{FTC}+\mathrm{EFV}$. la durée moyenne

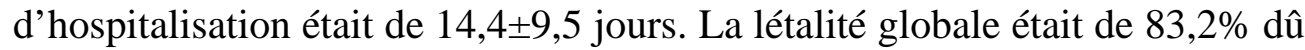
majoritairement à l'engagement cérébral (n=28; 33,3\%).

Conclusion. La prévalence et la mortalité des affections neuroméningées sur VIH sont élevées. Le retard diagnostic et la TNM influent sur le pronostic des patients, d'où l'intérêt d'un dépistage précoce de l'infection à VIH pour sa prise en charge afin de prévenir l’immunodépression.

Mots clés : Affections neuroméningées, VIH, prévalence, létalité, CHUBrazzaville 


\section{Introduction}

Les atteintes du système nerveux central(SNC) en rapport avec une infection par le virus de l’immunodéficience humaine(HIV) sont fréquentes et survienne en cas d’immunodépression avancée, le virus étant neurotrope (Cho, 1987. Moulignier, 2006). Au moins 75\% des examens post-mortem de cas de SIDA sont en rapport avec les infections opportunistes(OI) du SNC conséquence d'une déficience des mécanismes de défense immunitaire à médiation cellulaire (Françoise, 1989. Gray, 1988). La toxoplasmose cérébrale demeure encore la première IO la plus fréquemment rencontrée au cours du SIDA, suivi de la cryptococcose neuroméningée et des infections bactériennes (Kadjo, 2007. Demar, 2012. Fortes, 2011).L’infection à VIH devient aussi une maladie chronique et par conséquent le système nerveux fait face à l'infection chronique d'un virus neurotrope. Au Congo, la prévalence de l'infection à VIH est passée de 4,1 en 2003 à 3,2 en 2009(CREDES, 2004). Peu d'étude renseigne sur la prévalence des affections neurologiques chez les patients infectés par le VIH. Ce qui justifie l'objet de ce travail qui avait pour but la détermination de la prévalence des affections neuro-méningées au cours de l'infection à VIH couplé à la recherche des facteurs associés aux décès des patients admis en Maladies Infectieuses. Comme objectifs spécifiques ce présent travail visait à décrire les aspects épidémiologiques, cliniques et paracliniques de ces patients, décrire les aspects thérapeutiques et évolutifs et en fin déterminer les causes de décès des patients présentant une affection du SNC.

\section{Patients et Méthode}

\section{Type-cadre-période d'étude}

Etude rétrospective, analytique et descriptive des cas d'affections neuroméningées associées au VIH admis dans le service des Maladies Infectieuses du CHU de Brazzaville entre le $1^{\mathrm{er}}$ Janvier 2013 et le 31 mai 2015(soit 29 mois).

\section{Patients}

Etaient inclus les patients âgés d'au moins 17 ans chez lesquels le diagnostic d'une affection neuroméningée avait été confirmée, immunodéprimés par le VIH quelque soit le type, découverts en pré ou perhospitalisation, recevant ou non le traitement antirétroviral.

\section{Variables d'étude}

Epidémiologiques (âge, sexe, profession, lieu de résidence, statut matrimonial) cliniques( motif de consultation, délai de consultation, classification de l’OMS, type d'affection neuroméningée) Paracliniques (étude cyto-biochimique,bactériologique, parasitologique et mycologique du 
LCR, sérologie VIH, dosage des LTCD4, scanner cérébral avec et sans injection du produit de contraste, IDRT), Thérapeutiques(traitement anti infectieux, molécules antirétrovirales) et Evolutifs( guérison, rechute, , séquelles, décès, causes de décès).

\section{Analyse des données}

Les données ont été traitées au moyen du logiciel EPI info 3.3.2 (CDC Atlanta, USA) avec la détermination des statistiques descriptives et analytiques pour l'ensemble des sujets. Pour tous les tests, le seuil de signification a été fixé à 5\%.

\section{Résultats}

Cent cinquante dossiers inclus (10,5\% des admissions). Les patients avaient un âge moyen de 41,1 $\pm 11,8$ ans(extrême : 19 et 69 ans) de sexe féminin ( $n=86,57,3 \%$ ) et masculin( $n=64,42,7 \%$ ) avec un sex-ratio de 1,3.Ils étaient, sans emplois $(n=68 ; 45,3 \%)$, ouvriers $(n=30 ; 20,0 \%)$ résidant en ville ( $n=11275 \%)$,célibataires $(n=96 ; 64 \%)$ et mariés( $n=32 ; 21,3 \%)$, alphabétisés dans un tiers des cas.la contamination par le VIH1 était hétérosexuelle chez tous les patients(100\%). Le délai moyen de consultation était de 19,2 $\pm 1,3$ jours. La fièvre $(n=99 ; 66,0 \%)$, les troubles de la conscience $(n=66 ; 44,0 \%)$ et les crises convulsives $(n=37 ; 24,7 \%)$ étaient les principaux motifs de consultations (Tableau I). La majorité des patients $(\mathrm{n}=112 ; 74,7 \%)$ étaient au stade 4 de l'OMS. La raideur méningée (40\%), les signes focaux $(\mathrm{n}=62 ; 41,4 \%)$ étaient les principaux signes d'examen (Tableau II). L’aspect du LCR (Figure1) était clair ( $n=94$; 62,7\%), trouble ( $n=14 ; 9,3 \%)$, hyperproteinorachique (59,8\%), hypoglycorrachique $(43,8 \%)$.

La bactériologie isolait le germe dans 6 cas (Pneumocoque : 4 cas ; Méningocoque : 2cas) L'examen à l'encre de chine était positif dans 39 cas (34,8\%).La GERH était positive chez 35 patients.les anomalies scanographiques étaient dominées par les abcès $(n=12 ; 15,4 \%)$. Trente quatre patients était sous régime $\mathrm{TDF}+\mathrm{FTC}+\mathrm{EFV}$. la durée moyenne d’hospitalisation était de 14,4ะ9,5 jours. La létalité globale était de 83,2\% dû majoritairement à l'engagement cérébral $(n=28 ; 33,3 \%)$.Le stade II de l'OMS ( $\mathrm{p}=0,033)$, l'absence de traitement antirétrovirale $(\mathrm{p}=0,011)$,le retard à la consultation $(p=0,047)$ et la tuberculose neuroméningée $(p=0,000007)$ avaient influencé le pronostic chez nos patients (Tableau III).

\section{Discussion}

La présente étude malgré son caractère rétrospectif (Ouattara, 2007. Millogo, 2004) a pu lever le voile sur la prévalence et les facteurs associés au décès chez les patients infectés par le VIH, présentant une affection 
neuro-méningée dans le service des maladies Infectieuses du CHU de Brazzaville.

La prévalence était élevée dans notre étude comme retrouvé dans la littérature (Ki-Zerbo, 1999. Katlama, 1989) et ce, en rapport avec l'exposition des patients aux facteurs environnementaux. Les sujets jeunes adultes étaient les plus touchés dans notre série. Ils étaient majoritairement de sexe féminin, célibataires, en rapport avec les données épidémiologiques de la féminisation de l'infection à VIH. Il s'agissait aussi d'une population active sexuellement comme noté dans la plupart des données africaines (Kadjo, 2007. Millogo, 2004).La majorité de nos patients résidaient en milieux urbains qui sont des zones à forte densité de population, favorable à la contamination par l'infection à VIH. Quarante et huit de nos patients doublement infectés n’avaient pas d'emplois. Il s’agissait des ménagères. Cette couche sociale vulnérable est la plus exposée à l'infection par le VIH comme retrouvé dans la littérature(UNAIDS, 2013).L’infection par le VIH de type 1 était le principal terrain sous-jacent et dans la quasi-totalité des cas la transmission était faite essentiellement par voie hétérosexuelle.ces données sont conforme à l’épidémiologie du VIH dans nos régions(Millogo, 2004. UNAIDS, 2013).Au Vietnam, la consommation de drogue par voie injectable chez les jeunes de sexe masculin reste le principal mode de transmission du VIH (Rapport AIDS, 2001. Ngo, 2007).La fièvre, les troubles de la conscience ainsi que les céphalées avaient été les principaux motifs de consultation retrouvés chez nos patients comme noté dans les séries africaines (Ouattara, 2007.Millogo, 2004). Le délai moyen de consultation de nos patients paraissait long. Ces longs délais sont communs à plusieurs auteurs (Manga, 2009. Ouattara, 2007). Le dénie de la maladie à VIH propre aux populations étudiées, le bas niveau socio-économique et le recours aux traitements traditionnels justifie en partie ce retard à la consultation retrouvé en Afrique sub-saharienne. Les signes méningés et les signes focaux étaient les plus retrouvés à l'examen de l'appareil neurologique témoignant du stade avancé de l'état immunitaire des patients. En effet l'atteinte du système nerveux central au cours de l'infection par le VIH-1 est fréquente et est la conséquence soit de l'immunodépression induite par le virus, soit de l'atteinte du SNC par le virus lui-même, soit des effets secondaires liés aux traitements antirétroviraux dont la restauration immune et /ou la toxicité directe (Moulignier, 2006). L’aspect macroscopique du LCR était majoritairement clair chez nos patients ayant présenté une méningite lymphocytaire et dans 9,3\% des cas, ce liquide était trouble. Dans les méningites à germes pyogènes, le résultat de l'analyse du LCR est celui qui est décrit de façon classique (Ouattara, 2007). Ce pendant dans certaines méningites lymphocytaires on observe une discordance entre l'aspect macroscopique et les anomalies cyto-biochimiques comme décrit 
dans plusieurs séries africaines (Bissagnené, 1996. Kadjo, 2004. Soumaré, 2005. Ki-Zerbo, 1996). On a retrouvé une augmentation de la fraction protéique couplée à une hypoglycorachie dans la quasi-totalité des cas de tuberculose neuro-méningée comme signifié dans la littérature mais dans certains cas, devant les difficultés d'isoler le bacille de Koch à la bactériologie le traitement d’épreuve était utilisé comme argument d’orientation diagnostic (Soumaré, 2005. Eholié, 2009. Padrat, 1995. Bazin, 1995).Le diagnostic de la CNM avait été posé à l'examen direct à l'encre de chine comme décrit dans la littérature (Kadjo, 2004). Ce pendant d’autres examens plus spécifique comme la culture du LCR sur milieu de SABOURAUD sans actidione, l'Antigenemie cryptococcique ou la recherche de l'antigène cryptococcique dans le LCR qui auraient pu mettre en évidence les cryptocoques n’ont pas été réalisés faute de plateau technique. Cette difficulté est rencontrée dans plusieurs pays à ressources limitées (Millogo, 2004).le paludisme grave dans sa forme neurologique était diagnostiqué chez nos patients par la GERH plus frottis sanguin qui avait mis en évidence le plasmodium falciparum dans tous les cas, associé aux critères de gravité de l’OMS comme décrit dans la littérature, (Soumaré, 2007).Les images d'abcès,, une dilatation ventriculaire et les lésions ischémiques ont été les principales anomalies scanographiques retrouvées chez nos patients dans les proportions non négligeables. L’abcès cérébral, responsable d'un syndrome neurologique focal d'installation rapidement progressive dans un contexte d'hypertension intracrânienne était rattaché à la toxoplasmose cérébral comme signifié dans la littérature (Moulignier, 2006). les lésions ischémiques retrouvées dans notre série étaient en rapport avec les accidents ischémiques constitués(AIC) couronnant l'hypothèse selon laquelle, le risque d'accidents cérébrovasculaires est augmenté dans l'infection VIH-1(Moulignier, 2006).Par conséquent, la surveillance et la limitation des facteurs de risque vasculaire sont recommandées chez les PvVIH. La toxoplasmose cérébrale, la cryptococcose neuroméningée et la tuberculose neuroméningée étaient les différentes formes cliniques d'affections neurologiques les plus fréquemment rencontrées chez l'ensemble de nos patients. Ses résultats sont en conformité avec ceux obtenus dans plusieurs études africaines (Fortes, 2011. Ouattara, 2007. Soumaré, 2005). Le protocole associant Ténofovir, Entricitabine et Efavirenz était la trithérapie antirétrovirale la plus fréquemment prescrite chez nos patients, tenant compte des interactions médicamenteuses, de l’observance et du nombre de comprimés à prendre par jour comme recommandé par l'OMS (UNAIDS, 2014).Plus de la moitié de nos patient avait reçu une chimioprophylaxie au cotrimoxazole(43\%). L'efficacité de cette molécule est prouvée dans la prévention des infections opportunistes chez les patients ayant une immunodépression avancée. 
La durée moyenne d'hospitalisation de nos patients était longue comme noté dans d'autres séries africaines (Fortes, 2011. Ngo, 2007. Manga, 2009). Ces longues durées de prise en charge sont due en partie au fait que les patients consultent tardivement à un stade avancé de dépression immunitaire avec son corollaire l'apparition des infections opportunistes dont le coût du traitement reste à la charge des patients, prolongeant ainsi la durée d’hospitalisation. Notre étude retrouvait une létalité globale de 83,2\% due majoritairement à l'engagement cérébral, au choc anémique et à l'arrêt cardio-respiratoire. Il y avait une corrélation entre le degré d'immunodépression, le traitement antirétroviral et l'issue thérapeutique chez les malades. Le retard à la consultation $(\mathrm{p}=0,047)$, la tuberculose neuroméningée $(\mathrm{P}=0,0000007)$ avaient influencé de façon statistiquement significative la survenue de décès chez nos patients. Une étude réalisée au Vietnam montre que le degré d'anémie à l'admission est significativement associé à la mortalité à J21 de l'hospitalisation $(\mathrm{P}=0,029)$ et en fin d'hospitalisation $(\mathrm{P}=0,024)$ (Ngo, 2007). Ces données sont en accord avec la valeur pronostique péjorative de l'anémie au cours de l'infection à VIH (Diallo, 2003).

\section{Conclusion}

Les affectons neuroménigées au cours du VIH sont fréquentes avec une létalité élevée dans le service des Maladies Infectieuses du CHU de Brazzaville; ceci en rapport avec une immunodépression avancée. La prévention de ces affections passe par le dépistage et la prise en charge précoces de l'infection à VIH.

\section{Refrences:}

1. Cho ES, Sharer LR, Peress NS, Little B. Intimal proliferation of leptomeningeal arteries and brain infarcts in subjects with AIDS.J NeuropatholExpNeurol 1987 ; 46 :385(abstr.).

2. Moulignier A: Atteintes du système nerveux central et infection par le VIH-1. Rev neurol (paris) 2006 ; $162: 1,22-42$ )

3. Françoise Gray. Les lésions du système nerveux central au cours du SIDA. Médecine/sciences 1989; 5:145-151

4. Retrovirus in the nervous System. Proceedings of symposium sponsored by the National Institute of Health.Bethesda, 4-6 May 1987.Ann neurol 1988;23 (suppl):S1-217

5. Gray F, Gherardi R, Scaravilli F. the neuropathology of the acquired immune deficiency syndrome (AIDS): a review. Brain 1988;111:24566 
6. K.Kadjo, B.Ouattara, O.Kra et al. Toxoplasmose cérebrale chez le sideen dans le service de medecine interne du CHU de Treichville.Médecine d'Afrique Noire 2007 :54(1)

7. M.demar, D.Hommel, F.Djossou et al. Acute toxoplasmose in immunocompetent patients hospitalized in an intensive care unit in french Guiana. ClinMicrobiol Infect 2012; 18: E221-231

8. L.Fortes Déguénonvo, N.M.Manga, S.A.Diop , N.M.DiaBadiane, M.Seydi, C.T.Ndour, M.Soumaré, B.M.Diop, P.S.Sow.Profil actuel des patients infectés par le VIH hospitalizes à Dakar(Sénégal). Bull.Soc.Pathol.Exot.(2011)104:366-370

9. Centre National de la Statistique et des Etudes économiques(CNSSE) et Centre de recherche et d'étude en développement en santé publique(CREDES) : Evaluation national de la séroprévalence des infections à VIH et de la syphilis. Paris; 2004

10. B.Ouattara, S.P.Eholié, K.D.Adoubryn, O.Kra, H.Tia, C.G.KouadioYapo, V.Edo, J.Ouhon. Etude retrospective des meningitis bactériennes et à cryptocoques chez des sujets adultes infectés par le VIH à Abidjan(Côte d'Ivoire).Journal de Mycologie Médicale(2007)17,82-86.

11. A.Millogo, G-A.Ki-Zerbo,J.B.Andonaba et al. La Cryptococcose neuroméningée au cours de l'infection par le VIH au Centre hospitalier de Bobo-Dioulasso (Burkina Faso).Bull Soc PatholExot 2004,97,2,119-121.

12. Ki-Zerbo G, Sawadogo A, Millogo A, Andonaba J, Yameogo A, Ouedraogo I.

13. Manifestations neurologiques associees a l'infection par le VIH au Centre hospitalier de

14. Bobo-Dioulasso (Burkina Faso).BullSoc Path Exot 1999 ; 92 :23-6.

15. Katlama C. manifestations neurologiques au cours de l'infection à VIH. In :sida, infection à VIH: aspects en zone tropicale. Ellipses/Aupelf, 1989,129-140.

16. UNAIDS. Report on global AIDS Epidemic 2013. Geneva: UNAIDS; 2013

17. Ministry of health Vietnam. Rapport AIDS 2001.VNAIDS 2001.

18. A.T.Ngo, N.H.duc, N.H.Lan, M.Maynart, C.Mayaud, T.H.Quy. Evolutions fatales chez les patients vietnamiens co-infectés par le virus $\mathrm{VIH}$ et la tuberculose $\operatorname{BAAR}(+)$ au cours ou au décours immédiat de leur hospitalisation. RevPneumol Clin 2007 ; 63 :139146

19. N.M.Manga,S.A.Diop, C.T.Ndour et al. Dépistage tardif de l'infection à VIH à la clinique des maladies infectieuses de Fann, Dakar: circonstances de diagnostic, itinéraire thérapeutique des 
patients et facteurs déterminants. Médecine et maladies infectieuses 39(2009)95-100.

20. B.Ouatarra, S.P.Eholié, K.D.Adoubryn et al. Commentaires sur « Etude rétrospective des méningites bactériennes et à cryptocoques chez des sujets adultes infectés par le VIH à Abidjan ».Journal de Mycologie Médicale(2007) 17,87-88.

21. Bissagnené E., Domoua K. Situation actuelle des méningites, encéphalites infectieuses et suppurations intracrâniennes en zone tropicale. Med Afr Noire 1996 ; $43: 120-3$

22. Kadjo K.,Ouattara B., Niamkey EK. Intérêt de l'analyse du liquide céphalorachidien dans le diagnostic de la cryptococcose neuroméningée. Afrbiomed 2004 ;9:19-21.

23. M.Soumaré, M.Seydi, C.T.Ndour, Y.Dieng, N.F.Ngom-Faye, N.Fall, B.M.Diop. les méningites à liquide clair chez les patients infectés par le VIH à Dakar.Bull Soc PatholExot, 2005,98,2,104-107.

24. G.Ki-Zerbo, A.Sawadogo, A.Millogo, J.B.Andonaba, A.Yameogo, I.Ouedraogo, M.tamini, G.Durand. la cryptococcose neuro-méningée au cours du Sida : Etude préliminaire à l'hôpital de Bobo-Dioulasso (Burkina faso).Médecine d'Afrique Noire : 1996,43(1)

25. Eholié SP, Adou-Brynh D, Doumoua K, Kakoua, Ehuie, Gouamene A et al. Méningites lymphocytaires non virales de l'adule à Abidjan (Côte d'Ivoire). Bull Soc Path Exot 2009 ; 93(1) :50-54.

26. Padrat.F, Delattre J.Y. méningites chroniques-Editions techniquesEncycl, med.Chir .(paris-France), neurologie, 17-160-C, 1995,13p.

27. Bazin C. Méningite tuberculeuse : infection cérébro-méningée. Rev prat $1994 ; 44: 2214-2219$.

28. Soumaré Masserigne Seydi MoussaI, Diop Sylvie Audrey', FALL Naitssatou', NIAN Khadidiatou Naphte', Sidy A El Khalillshagh Cheikh 1, Dieng Yémou ,SOW Ahmad tyane', Diop Bernard MarceJ1, Sow Papa Salif. Epidémiologie, étiologie et pronostic des comas infectieux de l'adulte à Dakar (Sénégal). Rev. CAMES - Série A, Vol. 05,2007

29. UNAIDS, Report on Global AIDS Epidemic 2014.Geneva:2014

30. Diallo A, et al. Fréquence, facteur de risque et valeur pronostique de l'anémie associée au VIH/Sida chez l'adulte au Mali.Bull Soc Pathol Exot2003 ; $96: 123-7$ 
Tableau I. Répartition des patients selon le motif de consultation.

\begin{tabular}{llll}
\hline & $\mathbf{n}$ & $\mathbf{\%}$ \\
\hline Fièvre & 99 & $66,0 \%$ & \\
trouble de la conscience & 66 & $44,0 \%$ \\
Crises convulsives & 37 & $24,7 \%$ \\
Agitation psychomotrice & 26 & $17,3 \%$ \\
Céphalées + fièvre & 26 & $17,3 \%$ \\
Céphalées & 25 & $16,7 \%$ \\
Vomissements & 22 & $14,7 \%$ \\
Autres & 6 & $4,0 \%$ \\
\hline
\end{tabular}

Tableau II. Répartition des patients selon les signes d'examen neurologique.

\begin{tabular}{llll}
\hline Signes neurologiques & & $\mathbf{N}$ & $\mathbf{\%}$ \\
\hline & Glasgow $<8$ & 9 & $9,5 \%$ \\
Coma (n=95) & Glasgow $\geq 8$ & 86 & $90,5 \%$ \\
\hline & sous-total & 95 & $63,3 \%$ \\
\hline \multirow{3}{*}{ Signes de focalisation (n=150) } & Hémiparésie & 13 & $8,7 \%$ \\
& Tétraplégie & 3 & $2,0 \%$ \\
& Hémiplégie & 29 & $19,3 \%$ \\
& Atteinte des PC & 16 & $10,7 \%$ \\
& Autres & 1 & $0,7 \%$ \\
\hline \multirow{2}{*}{ Signes méningés (n=150) } & Raideur de la nuque & 60 & $40,0 \%$ \\
& Signe de Kernning & 21 & $14,0 \%$ \\
& Signe de Brudzinski & 16 & $10,7 \%$ \\
& Hyperesthésie cutanéé & 1 & $0,7 \%$ \\
\hline
\end{tabular}

Tableau III. Répartition des patients selon les facteurs associés au décès.

\begin{tabular}{cccccccc}
\hline FACTEURS & \multicolumn{3}{c}{ Décédé } & \multicolumn{3}{c}{ Non décédé } & \multicolumn{2}{c}{ Total } & \multirow{2}{*}{$\mathrm{P}$} \\
\cline { 2 - 6 } ASSOCIES & $\mathrm{n}$ & $\%$ & $\mathrm{n}$ & $\%$ & $\mathrm{n}$ & $\%$ & \\
\hline Age & & & & & & & \\
$18-29$ ans & 8 & $40,0 \%$ & 12 & $60,0 \%$ & 20 & $100,0 \%$ & 0,19 \\
$30-39$ ans & 26 & $56,5 \%$ & 20 & $43,5 \%$ & 46 & $100,0 \%$ & 0,92 \\
$40-49$ ans & 32 & $58,2 \%$ & 23 & $41,8 \%$ & 55 & $100,0 \%$ & 0,25 \\
$50-59$ ans & 12 & $57,1 \%$ & 9 & $42,9 \%$ & 21 & $100,0 \%$ & 0,9 \\
$60-69$ ans & 6 & $75,0 \%$ & 2 & $25,0 \%$ & 8 & $100,0 \%$ & 0,45 \\
\hline SEXE & & & & & & & \\
M & 40 & $62,5 \%$ & 24 & $37,5 \%$ & 64 & $100,0 \%$ & 0,22 \\
F & 44 & $51,2 \%$ & 42 & $48,8 \%$ & 86 & $100,0 \%$ & \\
\hline DÉLAI DE CONSULTATION & & & & & & \\
$<15$ jours & 13 & $39,4 \%$ & 20 & $60,6 \%$ & 33 & $100,0 \%$ & 0,047 \\
$\geq 15$ jours & 43 & $59,7 \%$ & 29 & $40,3 \%$ & 72 & $100,0 \%$ & 0,47 \\
Non precisé & 28 & $62,2 \%$ & 17 & $37,8 \%$ & 45 & $100,0 \%$ & 0,41 \\
\hline Coma & & & & & & & \\
Glasgow $<8$ & 7 & $77,8 \%$ & 2 & $22,2 \%$ & 9 & $100,0 \%$ & 0,28 \\
Glasgow $\geq 8$ & 53 & $61,6 \%$ & 33 & $38,4 \%$ & 86 & $100,0 \%$ & 0,28 \\
\hline STADE DE L'OMS & & & & & & & \\
II & 2 & $28,6 \%$ & 5 & $71,4 \%$ & 7 & $100,0 \%$ & 0,033 \\
III & 11 & $35,5 \%$ & 20 & $64,5 \%$ & 31 & $100,0 \%$ & 0,11
\end{tabular}




\begin{tabular}{cccccccc} 
IV & 71 & $63,4 \%$ & 41 & $36,6 \%$ & 112 & $100,0 \%$ & 0,44 \\
\hline $\mathrm{Hb}$ & & & & & & & \\
$<8 \mathrm{~g} / \mathrm{dl}$ & 48 & $94,1 \%$ & 3 & $5,9 \%$ & 51 & $100,0 \%$ & 0,94 \\
$\geq 8 \mathrm{~g} / \mathrm{dl}$ & 32 & $97,0 \%$ & 1 & $3,0 \%$ & 33 & $100,0 \%$ & \\
\hline $\mathrm{CD} 4(\mathrm{~mm} 3)$ & & & & & & & \\
$0-200$ & 81 & $58,3 \%$ & 58 & $41,7 \%$ & 139 & $100,0 \%$ & 0,06 \\
$201-350$ & 3 & $30,0 \%$ & 7 & $70,0 \%$ & 10 & $100,0 \%$ & 0,1 \\
$350+$ & 0 & $0,0 \%$ & 1 & $100,0 \%$ & 1 & $100,0 \%$ & 0,44 \\
\hline Chimio prophylaxie & & & & & & & \\
Oui & 27 & $48,2 \%$ & 29 & $51,8 \%$ & 56 & $100,0 \%$ & 0,18 \\
Non & 19 & $63,3 \%$ & 11 & $36,7 \%$ & 30 & $100,0 \%$ & 0,48 \\
\hline TARV & & & & & & & \\
Oui & 30 & $46,2 \%$ & 35 & $53,8 \%$ & 65 & $100,0 \%$ & 0,046 \\
Non & 53 & $66,3 \%$ & 27 & $33,8 \%$ & 80 & $100,0 \%$ & 0,011 \\
Non précisé & 1 & $20,0 \%$ & 4 & $80,0 \%$ & 5 & $100,0 \%$ & 0,23 \\
\hline
\end{tabular}

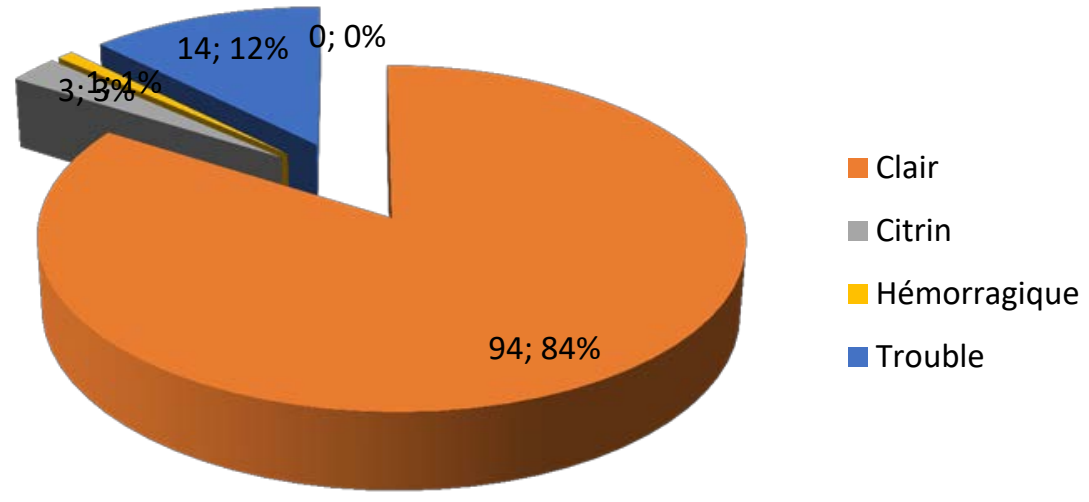

Figure 1. Répartition des patients selon l'aspect du LCR. 